\title{
Late Periprosthetic Joint Infection due to Staphylococcus lugdunensis Identified by Matrix-Assisted Laser Desorption/Ionisation Time of Flight Mass Spectrometry: A Case Report and Review of the Literature
}

\author{
Florian Szabados, ${ }^{1}$ Agnes Anders, ${ }^{1}$ Martin Kaase, ${ }^{1}$ Lennart Marlinghaus, ${ }^{1}$ \\ Sören G. Gatermann, ${ }^{1}$ Wolfram Teske, ${ }^{2}$ and Thomas Lichtinger ${ }^{2}$ \\ ${ }^{1}$ Institute for Hygiene and Microbiology, Department of Medical Microbiology, Ruhr-University Bochum, 44801 Bochum, Germany \\ ${ }^{2}$ Department for Orthopaedic Surgery, Ruhr-University Bochum, 44791 Bochum, Germany \\ Correspondence should be addressed to Florian Szabados, florian.szabados@rub.de
}

Received 21 December 2010; Revised 27 April 2011; Accepted 5 May 2011

Academic Editor: Jens Sondergaard

Copyright ( $) 2011$ Florian Szabados et al. This is an open access article distributed under the Creative Commons Attribution License, which permits unrestricted use, distribution, and reproduction in any medium, provided the original work is properly cited.

Staphylococcus lugdunensis, member to the group of coagulase-negative staphylococci, is previously thought to be rarely isolated. Recently other staphylococci have been described, which were supposedly related to S. lugdunensis, such as Staphylococcus pseudolugdunensis and Staphylococcus pettenkoferi. To decrease the rate misidentifications, an accurate identification method, such as matrix-assisted laser desorption ionization time of flight mass spectrometry or molecular methods, should be used. $S$. lugdunensis is usually associated with severe infections similar to those caused by $S$. aureus. Moreover, it has been described that skin infections due to $S$. lugdunensis are severely underreported and could be also underreported in periprosthetic joint infections. Ours is the first case of a late periprosthetic infection of the hip due to S. lugdunensis, identified by matrix-assisted laser desorption/ionisation time-of-flight mass spectrometry. A periprosthetic infection due to S. lugdunensis should be treated according to protocols of $S$. aureus periprosthetic infections, and therefore an accurate species identification is desirable.

\section{Introduction}

Staphylococcus lugdunensis has been described as a rare pathogen, which causes diseases similar to those caused by Staphylococcus aureus [1]. The reaction with pyrrolidonyl arylamidase (PYR) and ornithine decarboxylase (ODC) has been described to distinguish S. lugdunensis from other staphylococci [2]. Nevertheless, a PYR-negative $S$. lugdunensis isolate has been recently described [3], and it is likely that also ODC-negative S. lugdunensis exits. Moreover, species supposedly related to $S$. lugdunensis, such as Staphylococcus pseudolugdunensis [4] and Staphylococcus pettenkoferi [5, 6], have been described. S. lugdunensis, identified by superior methods, such as the recently described matrix-assisted laser desorption/ionisation time-of-flight mass spectrometry-(MALDI TOF MS-) based identification
[7], could be used to redefine the species of S. lugdunensis and newly investigate their prevalence and pathogenicity.

\section{Case Presentation}

A 47-year-old male patient was hospitalized because of severe pain and swelling of the right leg.

A cementless total hip arthroplasty was implanted two and a half years before, due to a previous osteonecrosis of the femoral head. In addition, the patient suffered from noninsulin-dependent diabetes mellitus type 2 and hepatitis B. The computer tomography (CT) showed a large cystic formation ventral to the hip reaching the psoas muscle (Figure 1). The CRP was elevated 23 -fold $(\mathrm{CRP}=116 \mathrm{mg} / \mathrm{L}$ ) and in the puncture S. lugdunensis was cultured from several 


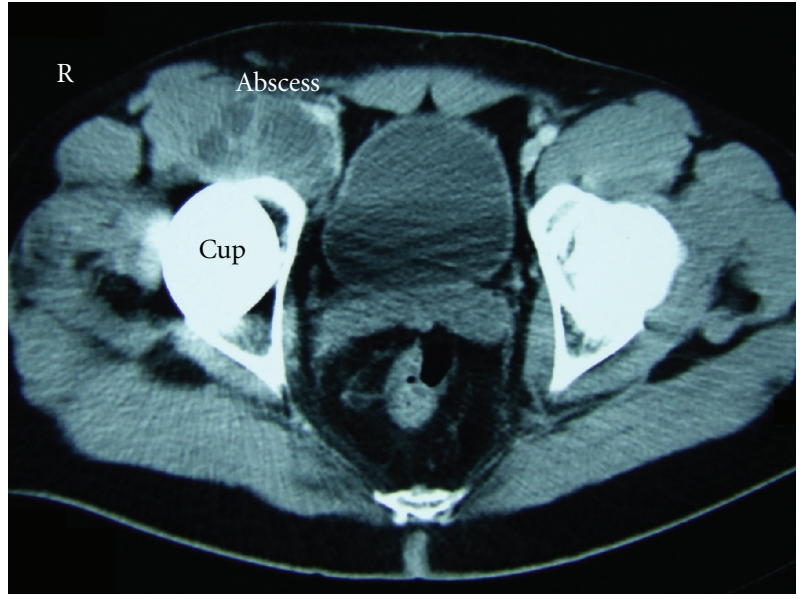

Figure 1: Abscess in front of the hip arthroplasty due to $S$. lugdunensis. The computer tomography (CT) shows a large cystic formation ventral to the hip reaching the psoas-muscle.

samples. CT-assisted drain was placed into the cyst and left for several days. A targeted antibiotic therapy with oxacillin $(2 \mathrm{~g}$ every $8 \mathrm{~h}$ ) and rifampicin (600 mg every $12 \mathrm{~h}$ ) was intravenously started and switched to oral application and maintained for two months. During the antibiotic therapy the cystic lesion was decreasing.

Two months later the patient complained again about pain in the right hip. Plain radiographs showed no signs of loosening of the cup or the femoral stem. The CRP was elevated with $76 \mathrm{mg} / \mathrm{L}$. A skeletal scintigram was performed. The enhancement of the synovia in the blood-pool phase and the increase of bone metabolism in the late phase of the bone scintigraphy were interpreted as a sign of a prosthetic infection. In the puncture of the joint itself S. lugdunensis was cultured as the causative pathogen again. In summary these results showed now a periprosthetic infection of the hip joint.

Therefore a two-staged revision of the prosthesis was performed. In the first stage, the prosthesis was explanted and replaced by a gentamicin-containing bone cement spacer into the acetabulum and a gentamicin-containing sponge into the femur. In an intraoperatively taken wound swab $S$. lugdunensis was cultured again. An antibiotic therapy was started with doxycycline (100 mg every $12 \mathrm{~h}$ ) and rifampicin (600 mg every $12 \mathrm{~h}$ ) and maintained for six weeks.

In the second stage four months later a conventional hip prosthesis was successfully reimplanted and doxycycline ( $100 \mathrm{mg}$ every $12 \mathrm{~h}$ ) and rifampicin (600 mg every $12 \mathrm{~h}$ ) were administered for two weeks again. Another four weeks later the prosthetic hip joint was revised because of recurrent dislocations of the head out of the cup. No bacteria were cultured from several intraoperatively taken wound swabs nor significant elevations of the CRP were observed. In a clinical follow-up 15 months later the patient showed a fair function of the hip. Plain radiographs and blood test results also showed no evidence of a periprosthetic reinfection.

2.1. Species Identification in Our Case. Staphylococci were primarily identified by typical colony morphology and

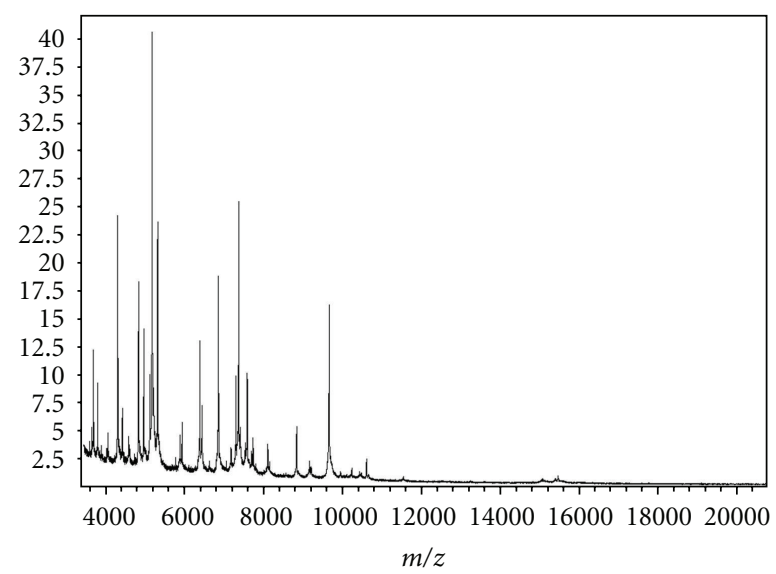

FIgURE 2: S. lugdunensis whole cell matrix-assisted laser desorption/ionisation time-of-flight MS fingerprint. Bacteria were covered with a layer of matrix ( $\alpha$-Cyano-4-hydroxy-cinnamic acid) and submitted for analysis. The proteomic fingerprint in the mass-tocharge ratio (Da) from $3000 \mathrm{Da}$ to $15.000 \mathrm{Da}$ was species specific and was matched to the Biotyper 2.0 database (Bruker Daltonics). Within a few minutes a highly accurate identification up to the species level was reported.

odor and were suspected as S. lugdunensis. Staphylococci were then misidentified by the GPI card by the Vitek-2 automated identification system (bioMérieux, Marcy l'Etoile, France). MALDI-TOF MS identified these staphylococci as S. lugdunensis (Figure 2). The species was confirmed by amplification of the species-specific tan $A$ and $f b l$ gene as previously described [7-9]. In vitro susceptibility testing was performed by the AST-580 card by the Vitek- 2 automated identification system (bioMérieux). An additional PCR was used to rule out the presence of a mecA gene.

\section{Discussion}

3.1. Importance of Accurate Species Identification in Periprosthetic Infections. In the few published literature concerning S. lugdunensis caused prosthetic infections mostly knee and other prosthetic sites rather than hip arthroplasties were included $[1,10]$. Up to now, data on periprosthetic hip infection due to $S$. lugdunensis is scarce. Within the group of CoNS, S. epidermidis has been reported to be an important pathogen in prosthetic joint infections. The contribution as a causative pathogen has been also discussed controversially [11], since S. lugdunensis is thought to be part of the normal skin flora, isolated primarily from lower abdomen and extremities [12]. An ica-dependent and an ica-independentbiofilms have been described, and these biofilms were discussed as a pathogenicity factor in biomaterial-associated infections [13]. Unexpectedly, data on pathogenicity factors of S. lugdunensis is scarce. Only a few reports have been described with regard to fibrinogenbinding adhesins $[14,15]$ and an $S$. lugdunensis synergistic hemolysin (SLUSH). The genome of a clinical strain of $S$. lugdunensis has been newly published and reveals a variety of pathogenicity factors such as the presence of a toxin 
pathogenicity island and further putative adhesins [16]. Notably, only $28 \%$ of the S. lugdunensis strains bind to fibrinogen [3], indicating differences between S. lugdunensis isolates with regard to binding to extracellular matrix proteins and their supposed pathogenicity. Since a long time S. lugdunensis is thought to be a rare but significant pathogen [1], but recently it was reported that skin and soft tissue infections due to S. lugdunensis are often underreported [17]. Typical morphological characteristics of this bacterium, such as a characteristic odor and a strong hemolysis after $48 \mathrm{~h}$ of incubation, have been described, which could help to increase the identification of S. lugdunensis [17]. In addition, the reaction with pyrrolidonyl arylamidase (PYR) and ornithine decarboxylase (ODC) has been described to distinguish S. lugdunensis from other staphylococci [2]. In contrast to this, a PYR-negative $S$. lugdunensis isolate has recently been reported [3], indicating that a significant amount of stains will be misidentified using the latter strategy. An ODC-negative S. lugdunensis has not been reported yet, but it is likely that such isolates also exit. Since $S$. lugdunensis usually is confirmed by a positive ODC reaction, previously identified collections likely do not contain an ODC-negative isolate per enclosure definition. Moreover, supposedly related new species have been described, such as S. pettenkoferi $[5,6]$ and S. pseudolugdunensis [4]. Some isolates of $S$. lugdunensis could be misidentified as other coagulase-negative staphylococci, especially when only a biochemical identification was performed.

Prosthetic joint infections can be classified in early infections, delayed infections, and late infections [18]. Early infections occur within three to four weeks. Delayed infections were defined as those up to 12 months, and late infections were defined as those occurring after more than 12 months [19]. Early and delayed infections are thought to be associated with pathogens introduced at the time of surgery, whereas late infections are discussed to be haematogenously acquired [19]. Staphylococci are most frequently isolated. For instance, coagulase negative staphylococci account for about $30 \%$ of infections in knee arthroplasty [20]. S. aureus is the most common haematogenously transmitted pathogen in all periprosthetic infections [19]. In many clinical microbiological laboratories coagulase negative staphylococci were identified by basic biochemical methods or supposedly specific reactions, such as PYR and ODC. A presumptive species identification using such methods is suitable for many staphylococcal species, but with an accuracy below of that of MALDI TOF MS and other molecular methods $[3,21]$. This lower accuracy, depending on the sample type, is usually believed to be sufficient. In case of prosthetic or periprosthetic infections due to suspected $S$. lugdunensis, these isolates should be confirmed by molecular methods $[8,9,22]$ or by MALDI TOF MS, which has been described as an easy-to-handle, fast, and reliable method for the identification of staphylococci $[7,21,23]$.

3.2. Microbiological Sampling and Surgical Management. One of the most important tests in the evaluation of a potential periprosthetic infection is culture of aspirated fluids and tissue samples. The positive predictive value of microbiologic culture is low, when performed in all patients before revision total hip arthroplasty, even when the clinical features did not necessarily suggest infection [24]. In a later study the positive predictive value of microbiologic culture was significantly higher in a collection of knee arthroplasties. This difference was discussed as a potential difference between knee and hip arthroplasties. On the other hand, the prevalence of infection in the second study was clearly higher (29\%) compared to the first study with $2 \%$. This also indicates that the positive predictive value of microbiologic culture is low, if solely used as a screening test for infection instead of as a confirmatory test for patients in whom clinical findings have raised the suspicion of infection [25]. When the culture results were correlated to the erythrocyte sedimentation rate or CRP, the sensitivity could be also increased [26]. In case of suspected periprosthetic infections it is recommended that five distinct intraoperative samples should be taken with separate instruments. An indistinguishable organism from at least three samples is strongly associated with infection. If a periprosthetic infection of the hip is diagnosed, the surgeon has to choose the optimal therapeutic strategy for the individual patient. For instance, limited surgical management involved debridement of a joint with exchange of modular components but retaining the prosthesis itself, combined with prolonged antibiotic therapy [19]. Prosthetic infections due to $S$. aureus seem to be associated with a higher rate of failure, when a limited strategy was compared to revision arthroplasty [27]. S. lugdunensis is often associated with severe clinical diseases similar to infections caused by S. aureus [1]. Therefore we performed a staged revision hip arthroplasty and not a limited surgical procedure in the reported case. Up to now, the treatment was successful, and there are no signs of a reinfection of the hip.

\section{Conclusion}

Periprosthetic hip infections due to $S$. lugdunensis are rarely reported. Depending on the identifying algorithm used in diagnostic laboratories, S. lugdunensis could be underreported or misidentified as other coagulase negative staphylococci. Identification of staphylococci using MALDI-TOF MS is straightforward, and the identification accuracy is equivalent to molecular methods. Therefore, these methods should be used for species identification of coagulase negative staphylococci rather than a previously described biochemical identification. S. lugdunensis is an important and often underestimated pathogen in severe skin and soft tissue infection, therefore, it seems to be likely that this pathogen is also underestimated in prosthetic and periprosthetic infections of the hip. Periprosthetic hip infections due to $S$. lugdunensis should be investigated in further detail to gain insights into the pathogenicity of this outstanding pathogen.

\section{Conflict of Interests}

The authors certify that there is no actual or potential conflict in relation to this paper. 


\section{References}

[1] K. L. Frank, J. L. Del Pozo, and R. Patel, "From clinical microbiology to infection pathogenesis: how daring to be different works for Staphylococcus lugdunensis," Clinical Microbiology Reviews, vol. 21, no. 1, pp. 111-133, 2008.

[2] T. Y. Tan, S. Y. Ng, and J. He, "Microbiological characteristics, presumptive identification, and antibiotic susceptibilities of Staphylococcus lugdunensis," Journal of Clinical Microbiology, vol. 46, no. 7, pp. 2393-2395, 2008.

[3] F. Szabados, Y. Nowotny, L. Marlinghaus et al., "Occurrence of genes of putative fibrinogen binding proteins and hemolysins, as well as of their phenotypic correlates in isolates of $S$. lugdunensis of different origins," BMC Research Notes, vol. 4, p. 113, 2011.

[4] Y. W. Tang, J. Han, M. A. McCormac, H. Li, and C. W. Stratton, "Staphylococcus pseudolugdunensis sp. nov., a pyrrolidonyl arylamidase/ornithine decarboxylase-positive bacterium isolated from blood cultures," Diagnostic Microbiology and Infectious Disease, vol. 60, pp. 351-359, 2008.

[5] K. Trulzsch, B. Grabein, P. Schumann et al., "Staphylococcus pettenkoferi sp. nov., a novel coagulase-negative staphylococcal species isolated from human clinical specimens," International Journal of Systematic and Evolutionary Microbiology, vol. 57, no. 7, pp. 1543-1548, 2007.

[6] C. Loïez, F. Wallet, P. Pischedda et al., "First case of osteomyelitis caused by 'Staphylococcus pettenkoferi', Journal of Clinical Microbiology, vol. 45, no. 3, pp. 1069-1071, 2007.

[7] F. Szabados, J. Woloszyn, C. Richter, M. Kaase, and S. Gatermann, "Identification of molecularly defined Staphylococcus aureus strains using matrix-assisted laser desorption/ionization time of flight mass spectrometry and the Biotyper 2.0 database," Journal of Medical Microbiology, vol. 59, no. 7, pp. 787-790, 2010.

[8] N. Noguchi, K. Goto, T. Ro et al., "Using the tannase gene to rapidly and simply identify Staphylococcus lugdunensis," Diagnostic Microbiology and Infectious Disease, vol. 66, pp. 120-123, 2009.

[9] E. M. Pereira, F. L. Oliveira, R. P. Schuenck, G. O. Zoletti, and K. R. Dos Santos, "Detection of Staphylococcus lugdunensis by a new species-specific PCR based on the fbl gene," FEMS Immunology and Medical Microbiology, vol. 58, no. 2, pp. 295298, 2010.

[10] N. B. Shah, D. R. Osmon, H. Fadel et al., "Laboratory and clinical characteristics of Staphylococcus lugdunensis prosthetic joint infections," Journal of Clinical Microbiology, vol. 48, no. 5, pp. 1600-1603, 2010.

[11] T. Hubiche, P. Del Giudice, and L. Roudière, "Staphylococcus lugdunensis in skin infections: pathogen or colonizing bacterium?" Journal of Clinical Microbiology, vol. 47, no. 9, p. 3057, 2009.

[12] L. Bieber and G. Kahlmeter, "Staphylococcus lugdunensis in several niches of the normal skin flora," Clinical Microbiology and Infection, vol. 16, no. 4, pp. 385-388, 2010.

[13] D. Mack, W. Fischer, A. Krokotsch et al., "The intercellular adhesin involved in biofilm accumulation of Staphylococcus epidermidis is a linear beta-1,6-linked glucosaminoglycan: purification and structural analysis," Journal of Bacteriology, vol. 178, no. 1, pp. 175-183, 1996.

[14] J. A. Geoghegan, V. K. Ganesh, E. Smeds, X. Liang, M. Höök, and T. J. Foster, "Molecular characterization of the interaction of staphylococcal microbial surface components recognizing adhesive matrix molecules (MSCRAMM) ClfA and Fbl with fibrinogen," Journal of Biological Chemistry, vol. 285, no. 9, pp. 6208-6216, 2010.

[15] M. Nilsson, J. Bjerketorp, B. Guss, and L. Frykberg, "A fibrinogen-binding protein of Staphylococcus lugdunensis," FEMS Microbiology Letters, vol. 241, no. 1, pp. 87-93, 2004.

[16] H. Tse, H. W. Tsoi, S. P. Leung, S. K. Lau, P. C. Woo, and K. Y. Yuen, "Complete genome sequence of Staphylococcus lugdunensis strain HKU09-01," Journal of Bacteriology, vol. 192, no. 5, pp. 1471-1472, 2010.

[17] S. Bocher, B. Tonning, R. L. Skov, and J. Prag, "Staphylococcus lugdunensis, a common cause of skin and soft tissue infections in the community," Journal of Clinical Microbiology, vol. 47, pp. 946-950, 2009.

[18] A. Trampuz and W. Zimmerli, "Prosthetic joint infections: update in diagnosis and treatment," Swiss Medical Weekly, vol. 135, no. 17-18, pp. 243-251, 2005.

[19] E. Moran, I. Byren, and B. L. Atkins, "The diagnosis and management of prosthetic joint infections," Journal of Antimicrobial Chemotherapy, vol. 65, supplement 3, pp. iii45-iii54, 2010.

[20] A. Stefánsdóttir, D. Johansson, K. Knutson, L. Lidgren, and O. Robertsson, "Microbiology of the infected knee arthroplasty: report from the Swedish Knee Arthroplasty Register on 426 surgically revised cases," Scandinavian Journal of Infectious Diseases, pp. 1-10, 2009.

[21] C. Dupont, V. Sivadon-Tardy, E. Bille et al., "Identification of clinical coagulase-negative staphylococci, isolated in microbiology laboratories, by matrix-assisted laser desorption/ionization-time of flight mass spectrometry and two automated systems," Clinical Microbiology and Infection, vol. 16, no. 7, pp. 998-1004, 2010.

[22] K. S. Chatzigeorgiou, N. Siafakas, E. Petinaki, and L. Zerva, "fbl gene as a species-specific target for Staphylococcus lugdunensis identification," Journal of Clinical Laboratory Analysis, vol. 24, no. 2, pp. 119-122, 2010.

[23] L. G. Harris, K. El-Bouri, S. Johnston et al., "Rapid identification of staphylococci from prosthetic joint infections using MALDI-TOF mass-spectrometry," International Journal of Artificial Organs, vol. 33, no. 9, pp. 568-574, 2010.

[24] R. L. Barrack and W. H. Harris, "The value of aspiration of the hip joint before revision total hip arthroplasty," Journal of Bone and Joint Surgery-Series A, vol. 75, no. 1, pp. 66-76, 1993.

[25] T. W. Bauer, J. Parvizi, N. Kobayashi, and V. E. Krebs, "Diagnosis of periprosthetic infection," Journal of Bone and Joint Surgery-Series A, vol. 88, no. 4, pp. 869-882, 2006.

[26] M. J. Spangehl, B. A. Masri, J. X. O’Connell, and C. P. Duncan, "Prospective analysis of preoperative and intraoperative investigations for the diagnosis of infection at the sites of two hundred and two revision total hip arthroplasties," Journal of Bone and Joint Surgery-Series A, vol. 81, no. 5, pp. 672-683, 1999.

[27] I. Byren, P. Bejon, B. L. Atkins et al., "One hundred and twelve infected arthroplasties treated with "DAIR" (debridement, antibiotics and implant retention): antibiotic duration and outcome," Journal of Antimicrobial Chemotherapy, vol. 63, pp. 1264-1271, 2009. 


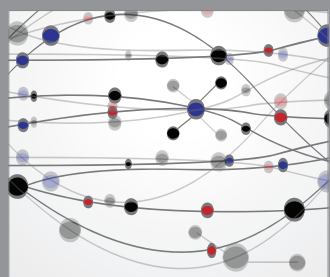

The Scientific World Journal
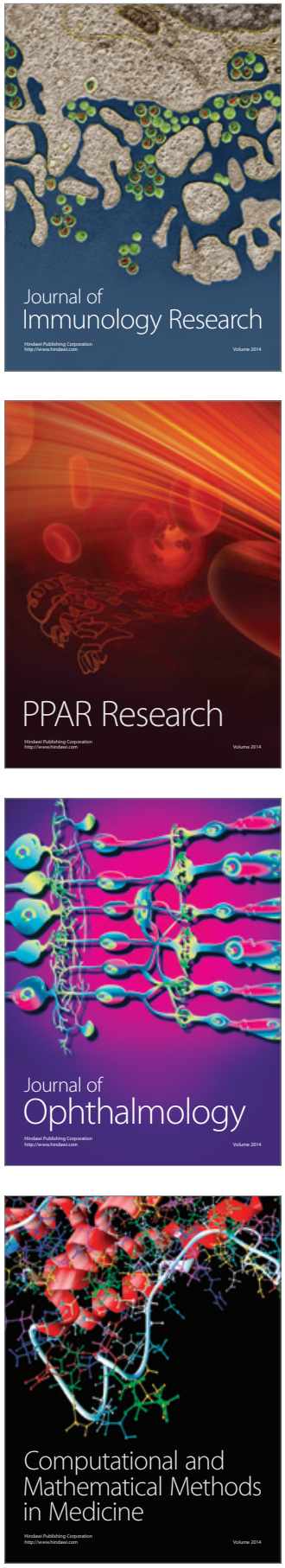

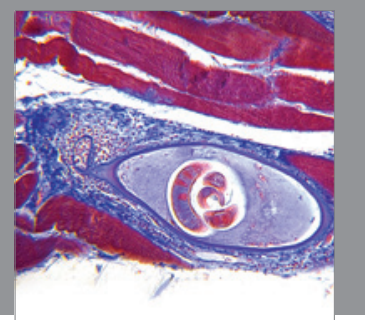

Gastroenterology

Research and Practice
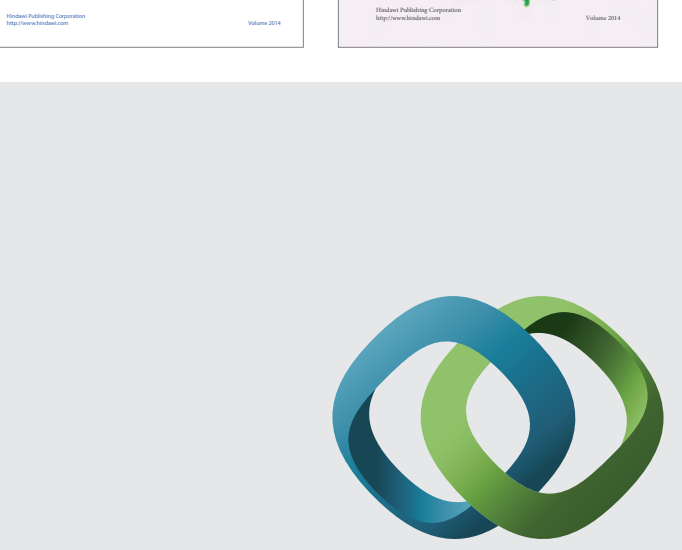

\section{Hindawi}

Submit your manuscripts at

http://www.hindawi.com
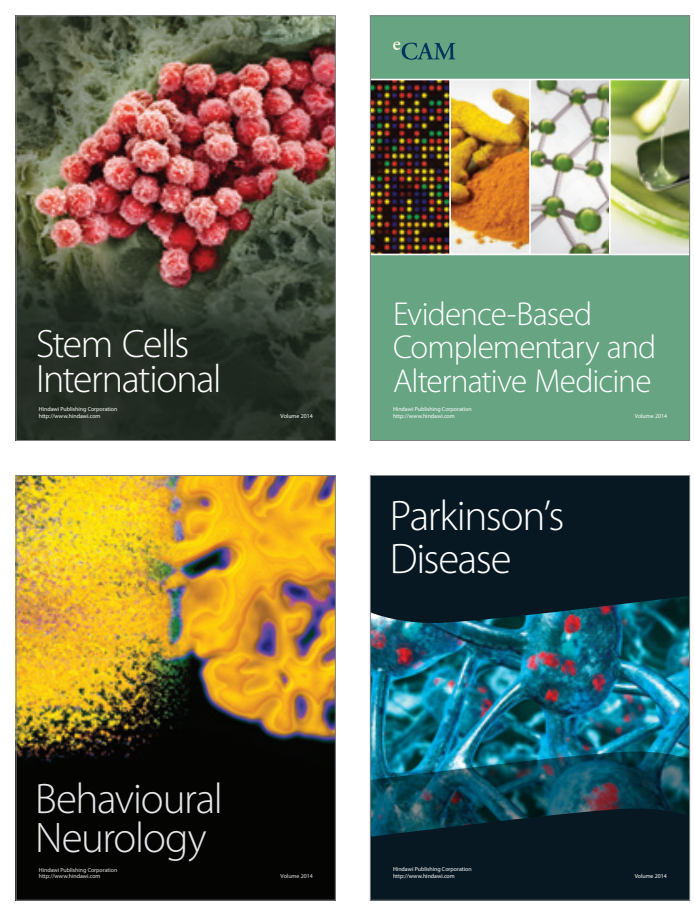

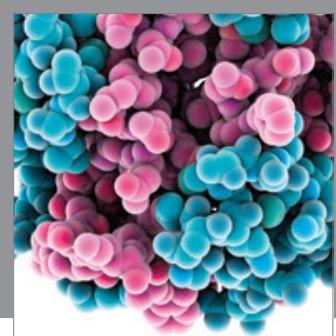

Journal of
Diabetes Research

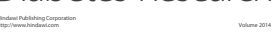

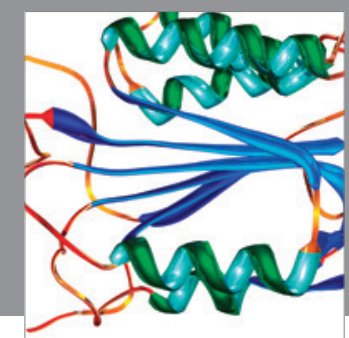

Disease Markers
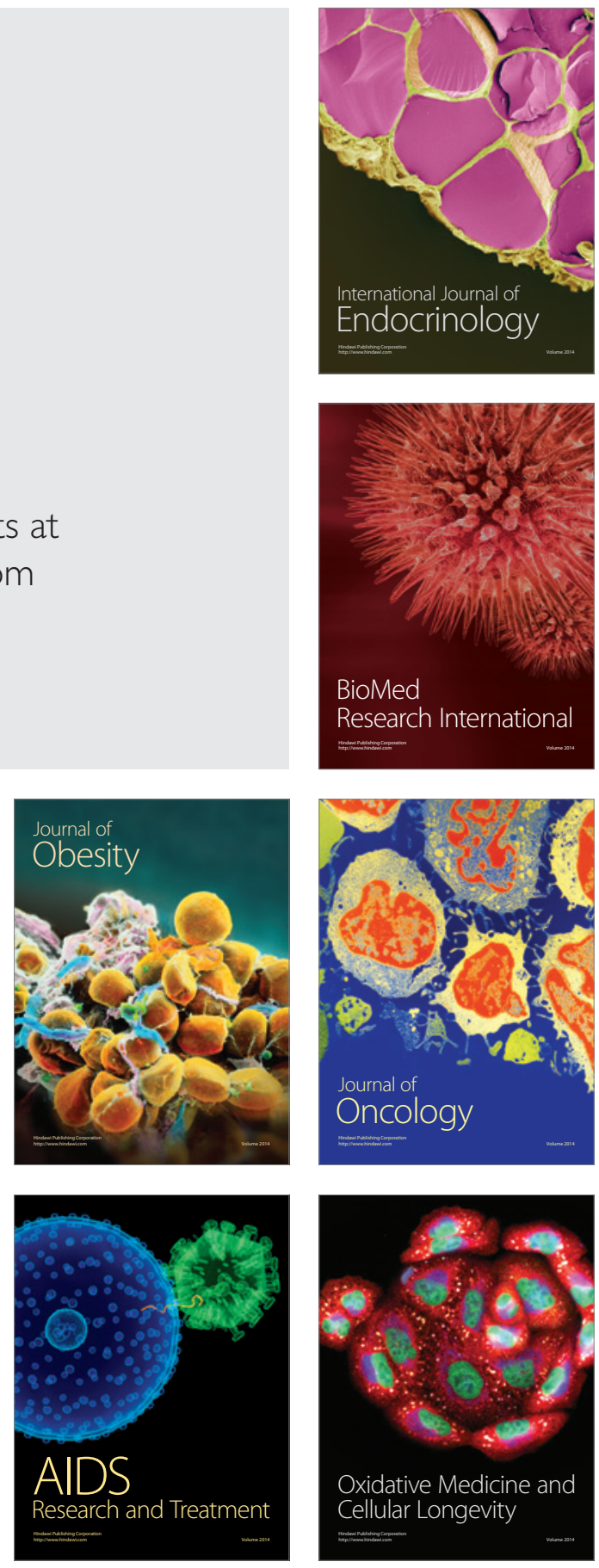